\title{
Tuberculosis contact tracing within a school environment: lessons for the future
}

\section{Pam Banner}

NSW Tuberculosis Program

Contact tracing plays a large role in the everyday work of chest clinics in NSW. It is a routine procedure that follows the 'concentric circle' method of screening. When a chest clinic becomes aware of a confirmed case of tuberculosis (TB), the inner ring of the circle (which comprises those with the highest risk of infection, including family and others in close contact with the index case) are screened first. If infection is detected in this group, screening proceeds to the medium-risk group and then, if necessary, to low-risk contacts, until no new infections are found. When explained, this method is usually readily understood and accepted by contacts. However, screening can become disrupted when contacts and others become fearful.

This paper highlights the case of a complex school screening which involved both screening all rings of the concentric circle (i.e. high, medium and low-risk contacts) and screening outside the circle (i.e. non-contacts).

In 2007 an overseas-born casual infants/primary school teacher in NSW was diagnosed with infectious cavitary TB disease by sputum smear, culture and chest X-ray. She had originally been diagnosed with pneumonia and was on sick leave for 1 month. One hour into the second day of her return to work, she had a massive haemoptysis (coughing up of blood) and was hospitalised.

Her household contacts were an overseas-born husband who was tuberculin skin test (TST) positive with a clear chest X-ray, and an Australian-born child who was TST negative. No other contacts were provided to the TB Coordinator at the Chest Clinic. Because of the teacher's smear and chest X-ray results, the TB Coordinator consulted with the then NSW Department of Health and local Public Health Unit (local units which work to identify, prevent and minimise public health risks to the community). Following this consultation, it was agreed to notify the school.

The school executive identified 160 children and staff the teacher had the closest contact with; they were included in the high-risk, first screening circle. A letter to parents, TB information, and a screening consent form were sent home with the identified children. The TB Coordinator gave an information session to staff, which was open to any concerned parents. It was decided that case management and screening would be performed at the school.

Initial screening identified one teacher and one child as infected. However, at follow-up screening of this group a few months later, 14 students, five teachers and the teacher's child returned positive TSTs. All had clear chest $\mathrm{X}$-rays and were offered preventive treatment through the Chest Clinic.

The Public Health Unit Director and TB Coordinator attended further meetings at the school. An information evening for parents was discussed but, at this time, the school executive declined. A further 40 medium-risk (second circle) students and teachers were then identified and screened, with another two students found to be infected.

As children began preventive treatment a rumour spread that there was an outbreak of TB at the school. Parents arriving at school were approached by media reporters. Screening was demanded by some parents whose children had never been in any of the teacher's classes.

An expert panel was convened to discuss the issue. Decisions were made to screen a low-risk group of a further 60 students and staff (the third circle); hold a parent information evening; release a statement to the media; send a letter to all local general practitioners (GPs) to inform them of the situation; and hold a meeting for GPs. Screening of this low-risk group found only one child infected. Eighteen non-contacts outside the circles were also screened at the request of parents, with no infected children found.

The TB Coordinator, along with representatives from the school, Public Health Unit and media representatives from the Local Health District (LHD), held a successful information evening for parents. It was stressed that there were no new cases of TB, was no ongoing risk of infection, those infected cannot spread TB to others and that $90 \%$ of people infected never develop disease.

From the 260 contacts screened, seven teachers, 18 students and the teacher's child were infected. This represented $12 \%$ of the high-risk group, $5.5 \%$ of the medium-risk group and $1.5 \%$ of the low-risk group. To date, no contacts have developed active disease. 
As a result of the screening exercise, there were valuable lessons learnt that can guide future school contact tracing exercises. It is recommended that after an initial teleconference with all relevant personnel (in this case, the TB Coordinator, Public Health Unit Director and school principal), the Public Health Unit Director (or equivalent) should write a briefing note for the relevant Health Department Director (in this case, the Chief Health Officer) and the head of the local health service (in this case, the Chief Executive of the LHD) including a summary of the key issues and recommendations. The media departments in both the LHD and the Health Department should also be alerted and updated.

The school principal, in conjunction with the local TB Coordinator, should hold an information session for all teachers at the school. This session should reinforce confidentiality issues and provide education on possible screening results. The school principal should send a letter and TB factsheet to parents of all children in the entire year(s) affected, even if only a small portion is to be screened. Also consider sending a letter to all parents in the school if the school is small. Education Department regional officers and school occupational health and safety regional officers should be informed by the school of the screening, and given a TB factsheet and an individualised screening plan for the event.

Advice should be given to the school executive to hold an open parent information evening before screening commences. The screening consent form should also request the details of the child's GP so he or she can be informed of the screening plan.

This example highlights the importance of implementing an agreed screening plan to effectively manage large screenings; this will ensure consistency of processes and information given and provide a central point of communication for all involved. 